ECT*-01-041; UTF/446

\title{
Spin force dependence of the parton distributions:
}

\author{
the ratio $F_{2}^{n}\left(x, Q^{2}\right) / F_{2}^{p}\left(x, Q^{2}\right)$ \\ Barbara Pasquini ${ }^{1,3}$, Marco Traini ${ }^{2,3}$, and Sigfrido Boffi ${ }^{4,5}$ \\ ${ }^{1}$ ECT* $^{*}$, Strada delle Tabarelle 286, I-38050 Villazzano (Trento), Italy \\ 2 Dipartimento di Fisica, Università degli Studi di Trento, I-38050 Povo (Trento), Italy \\ 3 INFN, Trento, Italy \\ ${ }^{4}$ Dipartimento di Fisica Nucleare e Teorica, Università degli Studi di Pavia, I-27100 Pavia, Italy \\ 5 INFN, Pavia, Italy
}

\begin{abstract}
Light-front Hamiltonian dynamics is used to relate low-energy constituent quark models to deep inelastic unpolarized structure functions of the nucleon. The approach incorporates the correct Pauli principle prescription consistently and it allows a transparent investigation of the effects due to the spin-dependent $S U(6)$-breaking terms in the quark model Hamiltonian. Both Goldstone-boson-exchange interaction and hyperfine-potential models are discussed in a unified scheme and a detailed comparison, between the two (apparently) different potential prescriptions, is presented.
\end{abstract}

PACS numbers : 12.39.-x, 13.60.Hb, 14.20.Dh 


\section{INTRODUCTION}

Constituent quark models (CQM) provide a basic tool for the description of low-energy hadron phenomena. Important features of non-perturbative quantum chromodynamics (QCD) can be incorporated in the CQM providing a framework for quantitative calculations of hadron properties and reaction observables. In particular CQM can be made to incorporate some of the basic symmetries [1] of QCD and, at the same time, can be defined within relativistic frameworks where covariance is preserved [2].

Gluons and quark-antiquark pairs surrounding a current quark are considered an integral part of it and together they make the constituent quark: a picture which seems to be substantiated by recent lattice QCD results [3].

Once explicit gluon degrees of freedom are integrated out, spin-dependent forces emerge within a potential description. Such forces between quarks have been associated to the chromomagnetic interaction of one-gluon exchange (OGE) [4], to Goldstone-boson exchange (GBE) in connection with the breaking of chiral symmetry [5], and to the effects of instantons [6]. At present all three models seem to have enough flexibility to approximate, to a large extent, existing data and the question whether they are just different ways of describing the same thing, remains unanswered. Actually the debate on the "defects" of the OGE as well as of the GBE is particularly active [7,8]. The investigation has been mainly confined to low-energy hadron excitation spectrum [9] and to the electroweak form factors of the nucleon [10]. In the present work we want to enlarge considerably the front of a possible comparison between the OGE and the GBE dynamical mechanisms considering the effects of the spin-dependent forces on the nucleon deep inelastic responses (structure functions).

Despite the fact that the subject has a rather long story, we can show that only at present we have the necessary tools to address the problem in a simple and transparent way and to elucidate the important role of spin-dependent forces on parton distributions. Let us begin summarizing existing approaches.

F.E. Close in 1973 [11] discussed the sensitivity of the ratio $F_{2}^{n} / F_{2}^{p}$ in relation with spin- 
dependent forces. He used a model of the nucleon where the nucleon first breaks up into a quark (which then interacts with the electromagnetic field) and a "quasi" particle called core. The core can have spin 0 or 1 and these two components have equal probability in a $S U(6)$ symmetric model only (where spin-dependent forces are neglected). He concluded that the predictions for the ratio $F_{2}^{n} / F_{2}^{p}$ are related to the high-momentum behaviour of the proton- $\Delta$ transition form factors.

Carlitz [12] investigated the $S U(6)$ symmetry breaking effects in deep inelastic scattering concluding that the mass difference between $N$ and $\Delta$ states of the $\underline{56}$ baryons implies that the ratio $F_{2}^{n} / F_{2}^{p}$ should approach $1 / 4$ as $x \rightarrow 1$. In a more recent work Close and Thomas [13] related the $\Delta-\mathrm{N}$ mass difference, to the ratio of neutron and proton inelastic structure functions and to deep inelastic polarization asymmetries.

Nathan Isgur [14 in a quite recent paper investigates the hyperfine-perturbed quark model to make predictions for the nucleon spin-dependent distribution functions and shows that precise measurements of the asymmetries $A_{1}^{p}(x)$ and $A_{1}^{n}(x)$ (in the valence region) can test the model and verify the "normal" behaviour of the valence-quark spin distribution at variance with the (sometimes) invoked "spin crisis". In the same paper Isgur critically discusses the history of the $S U(6)$-breaking effects with particular emphasis on the ratio $F_{2}^{n} / F_{2}^{p}$. In fact both papers of Close and Carlitz and Thomas studied the behaviour of the spin $1\left(\psi^{1}\right)$ and zero $\left(\psi^{0}\right)$ pair wave-functions separately; an approximated scheme which is not consistent with the Pauli principle unless $\psi^{1}$ and $\psi^{0}$ have very special properties under the permutation group in three dimensions. Isgur concludes that the situation remains rather unclear also because most authors have attempted "absolute" calculations of the structure functions with the unavoidable need of assumptions, approximations and "procedures". A criticism which involves also other calculations [15] 18].

Aim of the present paper is a close investigation of the relation between the $S U(6)$ breaking effects, responsible for the $\Delta$-N mass splitting and the charge radius of the neutron, and the ratio $F_{2}^{n} / F_{2}^{p}$. In our approach the Pauli principle is fully satisfied and we can compare effects of spin-dependent forces originating from both: chromomagnetic (hyperfine) 
OGE interaction and chiral symmetry motivated GBE mechanism. Because of the large debate on the subject, this last result is probably the most motivating one, and we show that the Pauli principle effect reveals to be of crucial importance in the discussion.

\section{PARTONS AND QUARKS}

Let us introduce the method we use to calculate the proton and neutron structure functions and its quite natural connection with the nucleon wave-function built in a given quark model.

The work by Glück, Reya and coworkers [19] has shown that, starting from a parton parametrization at a low resolution scale $\mu_{0}^{2}$, the experimental deep inelastic structure functions at high-momentum transfer can be reproduced and even predicted [20]. $\mu_{0}^{2}$ is evaluated by evolving back the second moment of the valence distribution to the point where it becomes dominant. The procedure, closely related to a suggestion due to Parisi and Petronzio [21], assumes that there exists a scale, $\mu_{0}^{2}$, where the short range (perturbative) part of the interaction is negligible, therefore the glue and sea are suppressed, and the long range (confining) part of the interaction produces a proton composed of three (valence) quarks only. Jaffe and Ross [22] proposed thereafter to ascribe the quark model calculations of matrix elements to the hadronic scale $\mu_{0}^{2}$. For larger $Q^{2}$ their Wilson coefficients will give the evolution as dictated by perturbative QCD. In this way quark models, summarizing a great deal of hadronic properties, may substitute low-energy parametrizations?.

Following such a path, a partonic description can be generated from gluon radiation even off a pure valence quark system, which can be used to evaluate the non-perturbative input occurring in the Operator Product Expansion (OPE) analysis of lepton-hadron scattering in QCD [23]. A systematic analysis shows that the approach can consistently be developed

\footnotetext{
${ }^{1}$ Let us stress that for the QCD evolution one needs matrix elements of the twist-two part of the current and not the calculation of the full response at low scale.
} 
at Next-to-Leading Order both for polarized and unpolarized structure functions, including non-perturbative contributions from the nucleon cloud [24] or from the partonic substructure of the constituent quarks [25]. In addition it can consistently be improved including relativistic covariance effects within the light-front Hamiltonian dynamics [26].

In the light-front description of deep inelastic scattering, the parton model is recovered, in the Bjorken limit, due to the dominance of short light-cone distances in the relevant Feynmann diagrams. As a consequence the partonic description can be developed in the rest frame of the hadron by using light-cone formalism. In particular the $i$-th parton distribution can be related to the light-cone momentum density[2

$$
q_{i}^{\uparrow(\downarrow)}\left(x, \mu_{0}^{2}\right)=\frac{1}{(1-x)^{2}} \int d^{3} k n_{i}^{\uparrow(\downarrow)}\left(\mathbf{k}^{2}\right) \delta\left(\frac{x}{1-x}-\frac{k^{+}}{M_{N}}\right),
$$

where $k^{+} / P^{+}=k^{+} / M_{N}=\left(\sqrt{\mathbf{k}^{2}+m_{i}^{2}}+k_{z}\right) / M_{N}$ is the light-cone momentum fraction of the struck parton in the rest frame, $M_{N}$ and $m_{i}$ are the nucleon and parton mass respectively and $n_{i}^{\uparrow}\left(\mathbf{k}^{2}\right), n_{i}^{\downarrow}\left(\mathbf{k}^{2}\right)$ represent the light-cone momentum density of the $i$-th parton whose spin is aligned $(\uparrow)$ or anti-aligned $(\downarrow)$ to the total spin of the parent nucleon. If one assumes that at the scale $\mu_{0}^{2}$ only the $u$ and $d$ constituent quarks are resolved, the momentum densities can be written

$$
n_{u(d)}^{\uparrow(\downarrow)}\left(\mathbf{k}^{2}\right)=\left\langle N, J_{z}=+\frac{1}{2}\left|\sum_{i=1}^{3} \frac{1+(-) \tau_{i}^{z}}{2} \frac{1+(-) \sigma_{i}^{z}}{2} \delta\left(\mathbf{k}-\mathbf{k}_{i}\right)\right| N, J_{z}=+\frac{1}{2}\right\rangle .
$$

The light-cone distributions (2) can be evaluated including relativistic effects as introduced by a light-front formulation of a three-body interacting system [26]. As a consequence we remain within a constituent picture where the partons in the rest frame are identified with three (constituent) quarks at the hadronic scale, and covariance requirement as well as Pauli principle are fulfilled.

Since the hadronic scale $\mu_{0}^{2}$ turns out to be very low $\left(\mu_{0}^{2} \sim(0.1-0.2) \mathrm{GeV}^{2}\right)$, close to the

\footnotetext{
${ }^{2} \mathrm{~A}$ formal derivation of Eq. (仼) can be found in the paper by Mair and Traini in ref. [24].
} 
constituent quark mass 3 , we assume that the constituent picture at this scale is represented by a constituent quark model, with parameters fixed to reproduce the basic features of the nucleon spectrum in the energy region $1-2 \mathrm{GeV}$. The constituent quark models we will make use of, in the present paper, are the Isgur-Karl model (IK) proposed long ago [27] and the GBE recently developed [9,10,28].

In particular the unpolarized valence parton distributions $q^{\uparrow}\left(x, \mu_{0}^{2}\right)+q^{\downarrow}\left(x, \mu_{0}^{2}\right)=$ $q_{V}\left(x, \mu_{0}^{2}\right)$, (with $\left.q \equiv u, d\right)$, at the hadronic scale are related to the scalar momentum densities $n_{u(d)}(\mathbf{k})^{2}$ (with $\left.\int d^{3} \mathbf{k} n_{u(d)}\left(\mathbf{k}^{2}\right)=2(1)\right)$ calculated making use of the wave-functions of the IK or GBE models with no free parameters. The dynamical effects due to the $S U(6)$-breaking terms in the quark Hamiltonian are entirely embedded in the momentum densities and no approximation is required. Details of the approach can be found in ref. [26].

\section{RESULTS AND DISCUSSION}

The results for the (twist-two part of) proton and neutron structure functions predicted by the two models, are shown in Figs. 1 both at the hadronic scale and experimental scale $Q^{2}=10 \mathrm{GeV}^{2}$. The $x$-dependence of the IK and GBE models differs quite substantially in the large $x$-region both at the hadronic and at the experimental scale. In particular the IK model lacks high- $x$ components: a result related to the lack of high-momentum components in the nucleon wave-function. The relativized nature of the GBE Hamiltonian reflects into a larger component of high momenta in the corresponding densities, an important ingredient to reproduce the behaviour of the structure functions at high- $x$ related to the presence of the $\sum_{i=1}^{3} \sqrt{\mathbf{k}_{i}^{2}+m_{i}^{2}}$ term in the Hamiltonian and therefore manifested in all the relativized models.

Before entering the discussion let us notice that the expected results for $F_{2}^{n}$ and $F_{2}^{p}$ can

\footnotetext{
${ }^{3}$ The actual value of the scale $\mu_{0}^{2}$ ranges from $0.1 \mathrm{GeV}^{2}$, if only valence quarks are considered, to $0.37 \mathrm{GeV}^{2}$ 24, when non-perturbative $q-\bar{q}$ pairs and gluons are included.
} 
be separated in two main regions. The low- $x(x \lesssim 0.3)$ region will be dominated by gluonic and sea partons. In the present calculation they are generated via bremsstrahlung radiation through renormalization group evolution. It is well known 24 that the inclusion of these hard partons is not sufficient to explain the absolute values of the structure functions at low- $x$ and soft components have to be added at the hadronic scale to approach the data. Therefore we do not consider our calculation to be quantitative for that region. At most we will obtain a qualitative description of the two structure functions.

In the region of large- $x(0.3 \lesssim x)$ the structure functions $F_{2}^{n}$ and $F_{2}^{p}$ are dominated by valence parton effects which are sensitive to the quark model wave-functions. It is just that region where the results for the ratio $F_{2}^{n} / F_{2}^{p}$ can become a specific test for the model wave-functions.

Our main results are presented in Figs. 2, and a few comments are in order:

i) Despite of the large sea and gluon contributions produced by QCD evolution (as illustrated in the Figs. $1 \mathrm{a}$ and $1 \mathrm{~b}$ ) the ratio $F_{2}^{n} / F_{2}^{p}$ is scarcely influenced by the perturbative QCD radiative effects, as expected. $F_{2}^{n}\left(x, \mu_{0}^{2}\right) / F_{2}^{p}\left(x, \mu_{0}^{2}\right) \approx F_{2}^{n}\left(x, Q^{2}=10 \mathrm{GeV}^{2}\right) / F_{2}^{p}\left(x, Q^{2}=\right.$ $10 \mathrm{GeV}^{2}$ ) for the whole range $0.3 \lesssim x \lesssim 0.7$. Such insensitivity is, in our case, an advantage. In fact the effects of QCD evolution largely cancel in the ratio $F_{2}^{n} / F_{2}^{p}$ compensating also the uncertainties coming from an evolution which starts from a quite low hadronic scalef. As a result, the details connected with the model wave-functions are emphasized (see Figs. 2 a,2 b).

ii) In particular it is evident that the Leading Order and the Next-to-Leading Order results do not differ appreciably.

iii) The ratio $F_{2}^{n} / F_{2}^{p}$ differs from the value $2 / 3$, the asymptotic value for $S U(6)$-symmetric models, because of the presence of spin-dependent forces in the model Hamiltonian.

\footnotetext{
${ }^{4}$ We do not need to enter a large discussion to justify our evolution approach because of such large cancellation. Details and discussions can be found in ref. [24].
} 
iv) Both the chromomagnetic (hyperfine) interaction of the IK model and the chiral mechanism of the GBE model fail to reproduce the correct behaviour of the experimental data in the region of validity of our calculation, namely $0.3 \lesssim x \lesssim 0.7$. A conclusion already known in the case of the IK model [24], but valid also for the GBE model. Note that this discrepancy is highly significant, since it concerns the region of Bjorken- $x$ where valence quarks dominate. Therefore complications arising from sea and gluon contributions cannot affect these results.

v) The effects of the large amount of high-momentum components generated by the relativistic expression of the kinetic energy can be noticed in the case of the GBE model, where the deviation with respect to the $S U(6)$-symmetric value $2 / 3$ is shifted at higher value of $x$. Similar effects are found in a calculation [29] which makes use of the relativized extension of the IK model proposed by Isgur and Capstick [30].

vi) It should be stressed that the disagreement emerges only if the Pauli principle effects are properly included. A simple model of the scattering involving separately the effects from an active quark plus a core of spin 1 and 0 quark pairs, would naturally reproduce the decreasing behaviour of the ratio $F_{2}^{n} / F_{2}^{p}$ as function of $x$ [1] [13].

vii) The disagreement with the experimental data does not depend on the details of the wave-function, but on the specific form of the $S U(6)$ breaking terms introduced in the model Hamiltonian. They are quite similar in the case of GBE and OGE models 31 despite of the different dynamical mechanisms invoked.

In conclusion, by means of a direct and transparent light-front calculation, we have shown that the ratio between the neutron and the proton structure functions is sensitive to the spin-dependent part of the quark-model Hamiltonian describing $S U(6)$-breaking effects at low hadronic energy. The correct theoretical behaviour of the ratio can be obtained only if the Pauli principle is preserved in the explicit calculation of the structure functions. Both hyperfine interactions, from the one-gluon-exchange potential model and from the spin dependent part of the Goldstone-boson interaction, cannot reproduce the large- $x$ behaviour 
of the data even at the qualitative level. The $S U(6)$-breaking mechanism seen in deep inelastic scattering seems to be different from the dynamical effects at low-energy, at least in the two cases we studied. Previous studies of unpolarized structure functions [24] show that a model able to invert the tendency of a ratio larger than $2 / 3$ in the large- $x$ region is the algebraic model proposed by Iachello [32. Despite the fact that the model is built in such a way that only the symmetry properties are retained and no dynamical mechanisms can be deduced, the parametrization which emerges is consistent with the data, in particular if the structure of the (effective) constituent quark is taken into account [25],33]. Work to

include the instanton dynamics and to enlarge the study of $S U(6)$ breaking effects into the region of polarized parton distributions is in progress.

\section{ACKNOWLEDGMENTS}

We acknowledge useful conversations with Sergio Scopetta, Silvano Simula, and Vicente Vento. We are grateful to Wolfram Weise for a careful reading of the manuscript and interesting comments. We also thank R.F. Wagenbrunn for providing us with the nucleon wave-functions in the GBE model. 


\section{REFERENCES}

[1] G. Dillon, and G. Morpurgo, Phys. Lett. B 481 (2000) 239; Erratum B 485 (2000) 429, and references therein.

[2] B.D. Keister and W.N. Polyzou, Adv. Nucl. Phys. 20 (1991) 225; F. Coester, Progr. Part. Nucl. Phys. 29 (1992) 1; J. Carbonell, B. Deplanques, V.A. Karmanov, and J.F. Mathiot, Phys. Rep. 300 (1998) 215; S.J. Brodsky, H.C. Pauli, and S.S. Pinsky, Phys. Rep. 301 (1998) 299.

[3] S. Aoki et al., Phys. Rev. Lett. 82 (1999) 4392; J.I. Skullerud, and A.G. Williams, Phys. Rev. D 63 (2001) 054508.

[4] A. De Rújula, H. Georgi, and S.L. Glashow, Phys. Rev. D 12 (1975) 147.

[5] L. Ya. Glozman, and D.O. Riska, Phys. Rep. 268 (1996) 264.

[6] E.V. Shuryak, Rev. Mod. Phys. 65 (1993)1; T. Schafer and E.V. Shuryak, Rev. Mod. Phys. 70 (1998) 323.

[7] N. Isgur, Phys. Rev. D 62 (2000) 054026.

[8] L. Ya. Glozman, nucl-th/9909021.

[9] L. Ya. Glozman, W. Plessas, K. Varga, R.F. Wagenbrunn, Phys. Rev. 58 (1998) 094030; Phys. Lett. B 516 (2001) 183. Z. Papp, A. Krassnigg, W. Plessas, Phys. Rev. C 62 (2000) 044004

[10] F. Wagenbrunn, S. Boffi, W. Klink, W. Plessas, M. Radici, Phys. Lett. B 511 (2001) 33; L. Ya. Glozman, M. Radici, R.F. Wagenbrunn, S. Boffi, W. Plessas, Phys. Lett. B 516 (2001) 183.

[11] F.E. Close, Phys. Lett. B 43 (1973) 422.

[12] R. Carlitz, Phys. Lett. B 56 (1975) 345. 
[13] F.E. Close, and A.W. Thomas, Phys. Lett. B 212 (1988) 227.

[14] N. Isgur, Phys. Rev. D 59 (1999) 034013.

[15] A. Le Yaouanc, L. Oliver, O. Péne, and J.C. Raynal, Phys. Rev. D 11 (1975) 680; 12 (1975) 2137; 13 (1976) 1519(E); 15 (1976) 844; 18 (1978) 1733.

[16] Z. Dziembowski, C.J. Martoff, and P. Zyla, Phys. Rev. D 50 (1994) 5613.

[17] X. Song, and J.S. McCarthy, Phys. Rev. D 49 (1994) 3169; C.J. Benesh, T.Goldman, and G. J. Stephenson, Phys. Rev. C 48 (1993) 1379.

[18] H.J. Weber, Phys. Rev. D 49 (1994) 3160.

[19] M. Glück, E. Reya, and A. Vogt, Z. Phys. C 53 (1992) 127; Phys. Lett. B 306 (1993); Z. Phys. C 67 (1995) 433.

[20] for a recent discussion cfr. M. Glück, E. Reya, and A. Vogt, Eur. Phys. J. C5 (1998) 461; Bodo L., E. Reya, Phys. Rept. 332 (2000) 1.

[21] G. Parisi, and R. Petronzio, Phys. Lett. B 62 (1976) 331.

[22] R.L. Jaffe, and G.C. Ross, Phys. Lett. B 93 (1980) 313.

[23] M. Traini, L. Conci, and U. Moschella, Nucl. Phys. A 544 (1992) 731; M. Ropele, M. Traini, and V. Vento, Nucl. Phys. A 584 (1995) 634. W.Melnitchouk, A.W.Schreiber, and A.W.Thomas, Phys. Rev. D 49 (1994) 1183; S. A. Kulagin, W. Melnitchouk, T. Weigl, W. Weise, Nucl.Phys. A 597 (1996) 515; K. Kusaka, G. Piller, A. W. Thomas, A. G. Williams, Phys. Rev. D 55 (1997) 5299; R. Jakob, P. J. Mulders, J. Rodrigues, Nucl. Phys. A 626 (1997) 937; D. I. Diakonov, V. Yu. Petrov, P. V. Pobylitsa, M. V. Polyakov, C. Weiss, Phys. Rev. D 56 (1997) 4069; V. Barone, T. Calarco, and A. Drago, Phys. Lett. B 390 (1997) 287; H. Weigel, L. Gamberg, H. Reinhardt, Phys. Rev. D 55 (1997) 6910 .

[24] M. Traini, V. Vento, A. Mair, and A. Zambarda, Nucl. Phys. A 614 (1997) 472; A. 
Mair, and M. Traini, Nucl. Phys. A 624 (1997) 564; A. Mair, and M. Traini, Nucl. Phys. A 628 (1998) 296.

[25] S. Scopetta, V. Vento, and M. Traini, Phys. Lett. B 421 (1998) 64; S. Scopetta, V. Vento, and M. Traini, Phys. Lett. B 442 (1998) 28.

[26] P. Faccioli, M. Traini, and V. Vento, Nucl. Phys. A 656 (1999) 400.

[27] N. Isgur, and G. Karl, Phys. Rev. D 18 (1978) 4187.

[28] R.F. Wagenbrunn, private communication, February 2001.

[29] S. Simula, private communication, March 2001; F. Cardarelli, S. Simula Phys. Rev. C $62(2000) 065201$.

[30] S. Capstick, and N. Isgur, Phys. Rev. D 34 (1986) 2809.

[31] For a discussion on the similarities of the OGE and GBE spin-dependent forces: F. Cano, and M. Traini, Phys. Rev. C 61 (2000) 065202.

[32] R. Bijker, F. Iachello, and A. Leviatan, Ann. Phys. (N.Y.) 236 (1994) 69.

[33] S. Scopetta, and V. Vento, private communication, July 2001.

[34] H.L. Lai, et al., hep-ph/9903282. 


\section{Figure captions}

Fig. 1 a: The proton structure function as predicted by the IK and GBE models at the hadronic scale (dotted and dot-dashed curves, respectively), and at the scale $Q^{2}=10$ $\mathrm{GeV}^{2}$ (dashed and continuous lines, respectively). Fit to the experimental data from the CTEQ5(D) analysis of ref. [34]: triangles.

Fig. 1 b: As in Fig. 1 a for the neutron structure function.

Fig. 2 a: The ratio $F_{2}^{n} / F_{2}^{p}$ as function of $x$ for the GBE model at the hadronic scale (dot-dashed curve), and at the scale $Q^{2}=10 \mathrm{GeV}^{2}$; Leading-Order evolution (dotted curve), Next-to-Leading-Order evolution (full curve). Fit to the experimental data from the CTEQ5(D) analysis of ref. [34]: triangles.

Fig. 2 b: The ratio $F_{2}^{n} / F_{2}^{p}$ as function of $x$ for the IK model. Notations as in Fig. 2 a. 


\section{FIGURES}

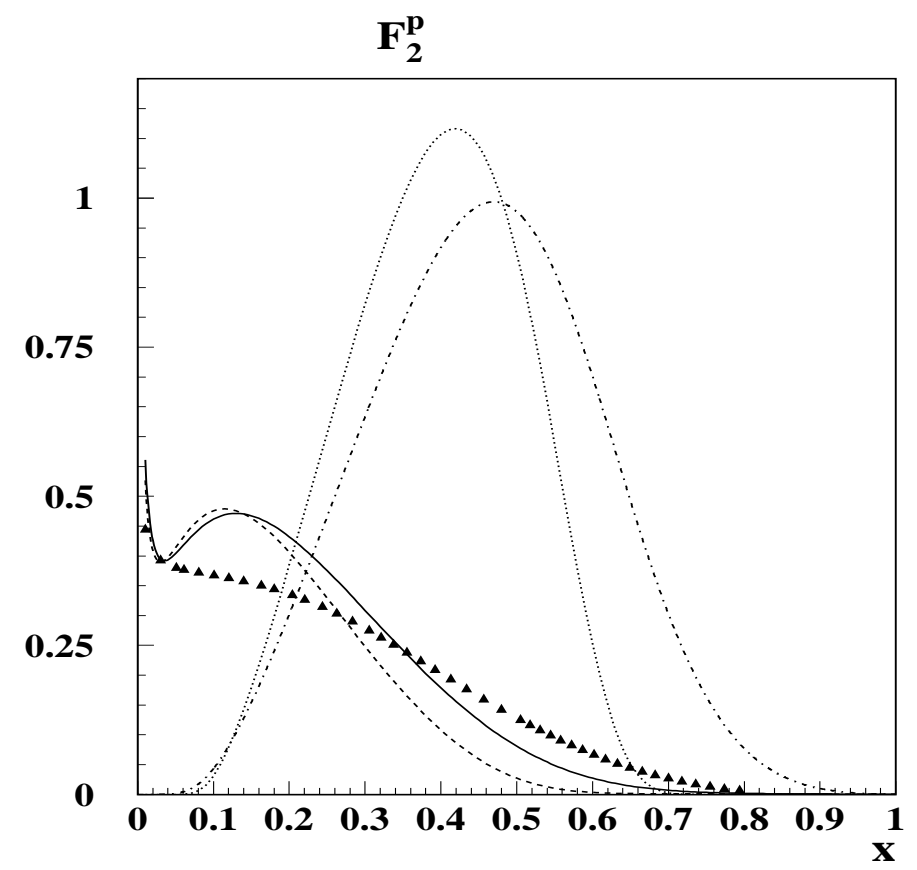

Figure 1 a

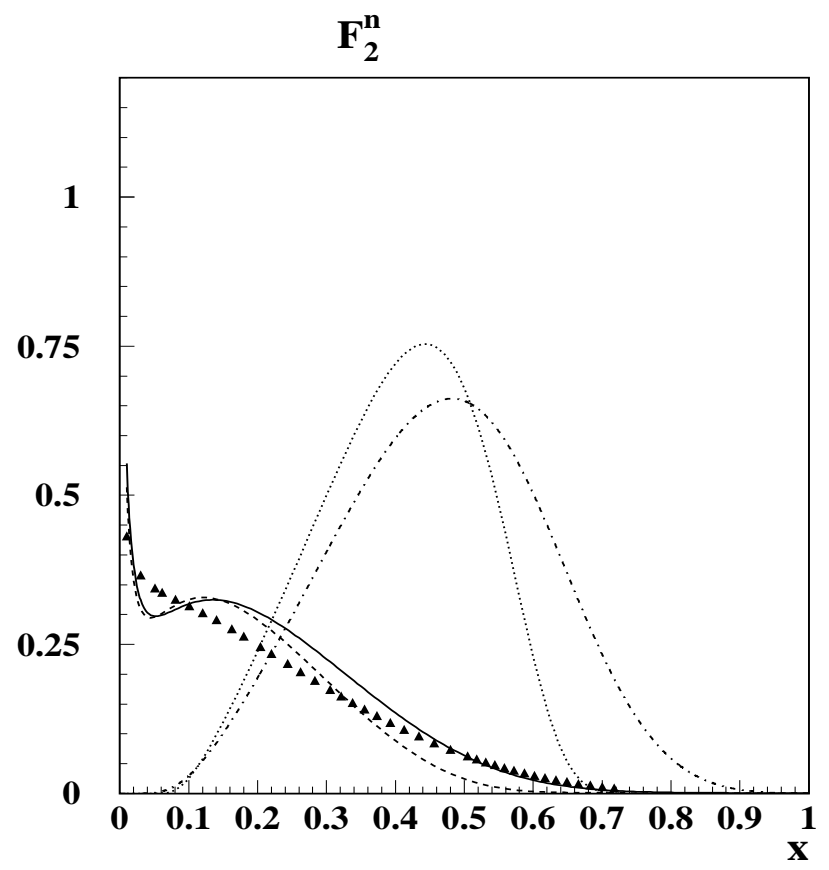

Figure $1 \mathrm{~b}$ 


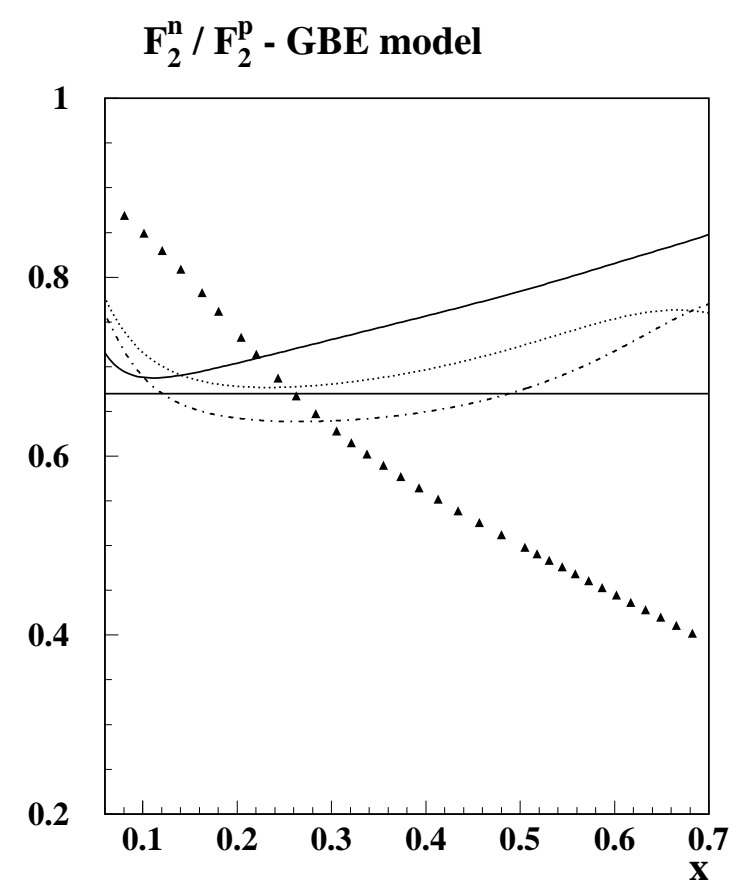

Figure 2 a

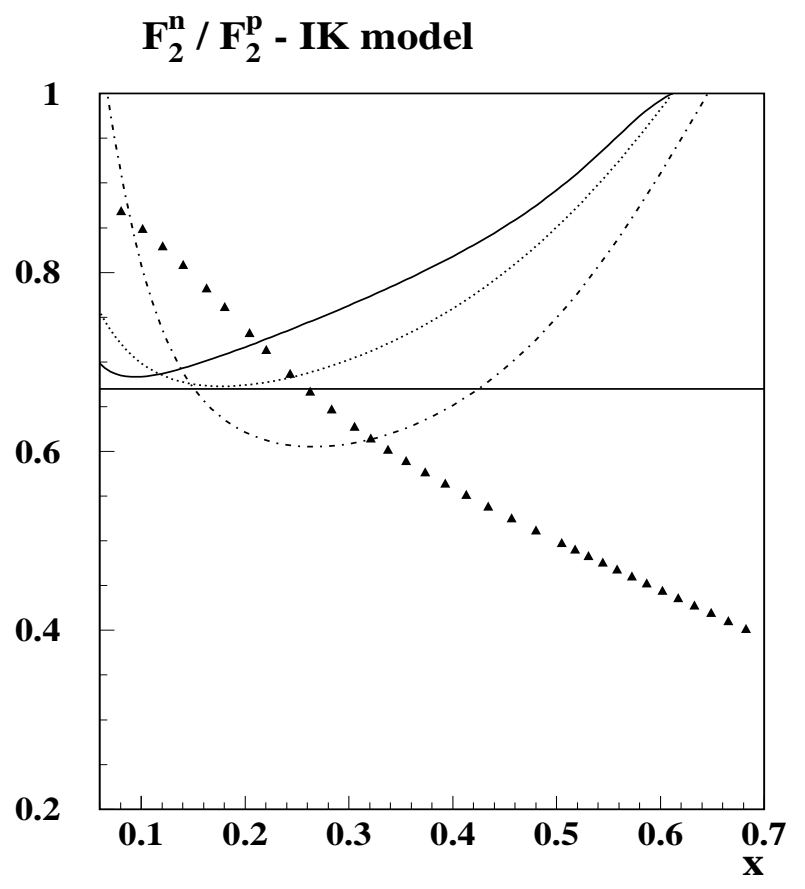

Figure $2 \mathrm{~b}$ 